\title{
Gerenciamento dos medicamentos em hospitalares públicos
}

\author{
Viviane Alves Miranda
}

Graduanda de Administração Hospitalar. Presidente de licitação da Prefeitura Municipal de Garanhuns. Email: vivialves_01@hotmail.com

\section{Vivianna Lins de Arruda}

Professora da Autarquia de Ensino Superior de Arcoverde - AESA.

Email: vivianne_arruda@hotmail.com

\section{Elisangela de Sousa Branco}

Doutora em Oceanografia Biológica pela Universidade Federal de Pernambuco - UFPE (2007) e mestre em Oceanografia Biológica pela Universidade Federal de Pernambuco. Professora efetiva da Autarquia de Ensino Superior de Arcoverde e da Autarquia de Ensino Superior de Garanhuns (2009).

Email: brancoesb@hotmail.com

RESUMO: A farmácia hospitalar é uma unidade clínica que, através do farmacêutico habilitado, busca garantir a qualidade de assistência aos pacientes fornecendo o uso de seus medicamentos. Por isto este estudo teve como objetivo mostrar a importância da farmácia hospitalar para os clientes/pacientes, tanto internos quanto externos em hospitais públicos, sabendo que a obtenção da cura ocorre através do correto tratamento medicamentoso. Esta pesquisa baseou-se em levantamento bibliográfico referente aos anos de 1998 a 2011 , enfatizando o gerenciamento de medicamentos em hospitalares públicos. A compra destes pode ser realizada através de várias maneiras, tais como, compra direta, licitação pública ou através de cooperativas visando economia de escala. A distribuição dos medicamentos para os pacientes é feita por dose única (paciente internado que recebe o medicamento por dosagem e no horário indicado na prescrição médica) e pela distribuição geral (pacientes externos que fazem a consulta e recebe o medicamento prescrito pelo médico para o tratamento por um mês ou até mesmo por mais tempo, e estão disponíveis nas farmácias públicas). Portanto, cabe ao administrador executar as tarefas de planejamento, controle e uma série de requisitos favoráveis a uma boa administração da farmácia hospitalar. Pois, atualmente ainda há muito para ser melhorado no gerenciamento e na distribuição dos medicamentos através dos órgãos públicos aos pacientes que deles necessitam.

PALAVRAS-CHAVES: farmácia hospitalar, medicamentos, farmácia pública.

\section{Management of medicines in public hospital}

\begin{abstract}
The hospital pharmacy is a clinical unit that, through the qualified druggist, it searches to guarantee the quality of assistance to the patients, supplying the use of its medicines. Because of this, this study it had as objective to show the importance of the hospital pharmacy for the patient/customers, as internal as external in public hospitals, knowing that the attainment of the cure occurs through the correct medicine treatment. This research was based on referring bibliographical survey to the years of 1995 to 2010, emphasizing the medicine management in publics' hospitals. The purchase of these can be carried through some ways, such as, direct purchase, public licitation or through cooperatives aiming at scale economy. The distribution of medicines for the patients is made by unique dose (patient interned that receives the medicine for dosage and in the schedule indicated in the medical lapsing) and by the general distribution (patient external that make the consultation and receive the medicine prescribed by the doctor for the treatment for one month or even for more time, and they are available in public pharmacies). Therefore, the administrator is supposed to execute the planning tasks, control and a series of requirements favorable to a good administration of the hospital pharmacy. Cause there is still a lot to be improved in the management and the distribution of medicines through the public agencies to the patients that need them.
\end{abstract}

KEY-WORDS: hospital pharmacy, medications, public pharmacy 


\title{
1. INTRODUÇÃO
}

A farmácia hospitalar é um órgão assistencial que desenvolve vários tipos de atividades, dentre elas estão: armazenamento, dispensação, controle e distribuição de medicamentos e correlatos. É de inteira responsabilidade na orientação de pacientes internos e ambulatórios, propondo sempre a eficácia da terapêutica, como também da redução de custos.

Cavallini e Bisson (2002, p. 22) definem:

\begin{abstract}
A Farmácia Hospitalar é uma unidade clinica de assistência técnica e administrativa, dirigida por farmacêutico, integrada funcional e hierarquicamente às atividades hospitalares, tendo como principal função garantir a qualidade de assistência prestada ao paciente por meio do uso seguro e racional de medicamentos e correlatos, adequando sua aplicação à saúde individual e coletiva, nos planos assistencial, preventivo, docente e investigativo, devendo, para tanto, contar com farmacêuticos em número suficiente para o bom desempenho da assistência.
\end{abstract}

Portanto, a farmácia hospitalar é uma unidade clínica que, através do farmacêutico habilitado, busca garantir a qualidade de assistência aos pacientes fornecendo o uso de seus medicamentos, e é de grande apoio nos programas de saúde pública.

Segundo Simonetti; Novaes; Afonso (2009) a farmácia hospitalar é atualmente uma unidade do hospital que tem, dentre outros objetivos, garantir o uso seguro e racional dos medicamentos prescritos e responder à demanda de medicamentos dos pacientes hospitalizados.

A principal função da farmácia hospitalar é servir ao paciente atendendo suas necessidades e oferecendo um serviço de qualidade, mas para a realização dessa proposta é necessário que o administrador esteja sempre atualizando seus conhecimentos, pois, é através do administrador que são executados as tarefas de planejamento, controle e uma série de requisitos favoráveis a uma boa administração.

Vale salientar que, para haver uma qualidade adequada nos serviços prestados através da farmácia hospitalar e um excelente desenvolvimento nos seus serviços através dos medicamentos e correlatos, sua estrutura organizacional deve ser bem elaborada e suas funções bem definidas.

Os recursos orçamentários/financeiros utilizados para manutenção de um hospital representam um percentual muito grande referente à aquisição de medicamentos numa farmácia hospitalar, para assim oferecer aos usuários uma atenção farmacêutica de qualidade e segura. Ainda de acordo com Eickhoff; Heineck; Seixas (2009) a racionalização dos gastos com medicamentos garante que o usuário receba a melhor terapêutica ao menor custo. É importante ressaltar que os medicamentos representam uma das maiores parcelas dos custos hospitalares, comprar bem é um dos principais meios para redução dos custos, isso significa verificar cuidadosamente, preços, prazo de entrega e qualidades dos medicamentos, onde se pode estabelecer um sistema racional de aquisição de medicamentos.

Em 30 de dezembro de 2010, o Ministério da Saúde aprovou a Portaria de $n^{\circ} 4.283$, que estabelecem diretrizes e estratégias para organização dos serviços de farmácia hospitalar.

Portanto, o objetivo desta pesquisa foi mostrar a importância da farmácia hospitalar para os clientes internos e externos de um hospital, mesmo sendo o setor que tem mais gastos no seu orçamento, mas de inteira responsabilidade para o bom funcionamento da entidade.

\section{METODOLOGIA}

Trata-se de um levantamento bibliográfico em artigos científicos entre os anos de 1998 a 2011 , enfatizando o gerenciamento de medicamentos em hospitais públicos. O primeiro capítulo abordará a aquisição de medicamento enfatizando as diferentes formas de se comprar os mesmos. No segundo capítulo será abordado a farmácia hospitalar, que consiste em suas funções e funcionalidade. No capítulo seguinte abarcará a distribuição dos medicamentos de acordo com as legislações vigentes. $\mathrm{E}$ por fim será discutida a distribuição dos medicamentos aos pacientes internados na rede hospitalar bem como para os pacientes externos. 


\section{AQUISIÇÃO DE MEDICAMENTOS}

A aquisição de medicamentos é uma das principais atividades da Gestão da Assistência Farmacêutica e deve estar estreitamente vinculada às ofertas de serviços e à cobertura assistencial dos programas de saúde. Uma boa aquisição de medicamentos deve considerar primeiro o que comprar (seleção); quando e quanto comprar (programação); e como comprar. O monitoramento e a avaliação dos processos são fundamentais para aprimorar a gestão e intervir nos problemas (MINISTÉRIO DA SAÚDE, 2006).

É muito complexo um processo de compra de medicamentos no setor público e abrange um conjunto de exigências administrativas e legais que devem ser cumpridas. A compra destes pode ser realizada através de várias maneiras, tais como, compra direta, licitação pública ou através de cooperativas visando economia de escala. A aquisição de medicamentos, na maioria das unidades de saúde, é feita através da própria unidade, podendo ser feita diretamente pelo serviço de farmácia, pela gerência, administração ou direção da unidade.

No setor privado, com uma política de compras de medicamentos definida, se consegue gerenciar bem o processo negociando com facilidade com seus fornecedores, criando um movimento constante de aquisição indispensável à manutenção dos estoques nas unidades.

Já no setor público, nota-se a dificuldade para a aquisição de quaisquer produtos e/ou serviços; pois tem que obedecer ao que rege a Lei $n^{\circ} 8.666 / 93$, fazendo com que os processos de compra fiquem demorados, tornando mais caros os próprios medicamentos, que são oferecidos aos órgãos públicos com os custos da máquina burocrática introduzidos.

$\mathrm{Na}$ área de compras de um hospital, sempre terá que ter eficiência na sua organização, pois é de onde serão realizadas suas compras e sua distribuição dentro do hospital e tem que ser de maneira correta, pois este departamento onde irá realizar esses serviços estará trabalhando para a recuperação da saúde dos seus clientes/pacientes.

Para Gonçalves (2006) a forma de adquirir materiais e medicamentos em caso de urgência, poderá ser efetuada por meio de remanejamento entre os hospitais da rede, a título de empréstimos, enquanto que no caso de emergência a aquisição poderá ser efetuada por meio de compra no local, utilizando os recursos do caixa existente, com autorização dentro dos limites e competências estabelecidas.

Ao receber os medicamentos a administração deve promover a realização de exames, testes e verificações necessárias observando se está de acordo com o estabelecido pela administração, pois quando se trata de hospitais têm que ser mais rigoroso com a fiscalização, pois é a vida humana que se está em pauta.

Segundo Maximiano (2007, p. 57), "A administração é uma realização comum a todos os empreendimentos, seja ela, nos negócios, no governo ou na família, que sempre precisa de algum grau de organização, planejamento, liderança e controle".

A administração de materiais no setor público objetiva a compra e serviços com baixo custo na aquisição, no armazenamento e na distribuição, com sua devida qualidade, conformidade e continuidade, os objetivos de uma unidade de gerenciamento de materiais em hospitais visam garantir a continuidade da oferta dos seus serviços de saúde; seu baixo custo de aquisição, de realização do pedido e de manutenção dos estoques; alta rotatividade dos estoques; qualidade no atendimento, dos materiais; o bom relacionamento com os fornecedores e a obtenção do máximo retorno.

E de acordo com Silva-Neta; Branco; Leite (2011) para o atendimento das atividades hospitalares é necessário evitar o excesso e a falta de medicamentos, que são duas situações prejudiciais ao bom desempenho da organização, pois geram gastos adicionais que não agregam valor aos serviços e nem qualidade na prestação de serviços.

Por isto, o principal objetivo da administração é gerenciar, coordenar e controlar suas compras para que com isso exista controle no abastecimento e que não aconteça paralisação de atendimento médico por falta de medicamentos e correlatos. A administração de compras tem sua importância no que diz respeito à aquisição de todo material seja ele qual for, para que o hospital tenha uma boa execução em todos seus serviços prestados e seu gerenciamento seja bem executado.

O Art. $3^{\circ}$, $\S 1^{\circ}$ ao $4^{\circ}$, da Lei $9.787 / 99$, fala a respeito da obrigatoriedade do SUS em adquirir medicamentos genéricos: 
Art. 3o As aquisições de medicamentos, sob qualquer modalidade de compra, e as prescrições médicas e odontológicas de medicamentos, no âmbito do Sistema Único de Saúde - SUS, adotarão obrigatoriamente a Denominação Comum Brasileira (DCB) ou, na sua falta, a Denominação Comum Internacional (DCI).

- § 1 O O órgão federal responsável pela vigilância sanitária editará, periodicamente, a relação de medicamentos registrados no País, de acordo com a classificação farmacológica da Relação Nacional de Medicamentos Essenciais - RENAME vigente e segundo a Denominação Comum Brasileira ou, na sua falta, a Denominação Comum Internacional, seguindo-se os nomes comerciais e as correspondentes empresas fabricantes.

- § 2o Nas aquisições de medicamentos genéricos, quando houver, terá preferência sobre os demais em condições de igualdade de preço.

- § 30 Nos editais, propostas licitatórias e contratos de aquisição de medicamentos, no âmbito do SUS, serão exigidas, no que couber, as especificações técnicas dos produtos, os respectivos métodos de controle de qualidade e a sistemática de certificação de conformidade.

- $\S 4^{\circ}$ A entrega dos medicamentos adquiridos será acompanhada dos respectivos laudos de qualidade.

\section{FARMÁCIA HOSPITALAR}

A Farmácia Hospitalar brasileira é regulamentada pela Resolução n 492 de 26 de novembro de 2008, do Conselho Federal de Farmácia (CFF), esta substitui a anterior, de número 300 de 1997. O Ministério da Saúde, recentemente publicou a portaria $n^{\circ} 4283$ de 30 de dezembro de 2010, que estabelecem diretrizes e estratégias para organização das Farmácias Hospitalares. Um marco regulatório.

Aos poucos, a farmácia hospitalar vem assumindo sua identidade própria, a partir de modelos europeus (espanhóis) e do modelo americano (Pharmaceutical Care), isso é bastante positivo, pois tem melhorado a cada dia a qualidade deste serviço.

Farmácia hospitalar é atualmente uma unidade do hospital que tem, dentre outros objetivos, garantir o uso seguro e racional dos medicamentos prescritos e responder a demanda de medicamentos dos pacientes hospitalizados. (SIMONETTI; NOVAES; AFONSO, 2009).

A farmácia hospitalar como unidade técnico-administrativa é de âmbito assistencial, ligada à direção do hospital e integrada com as demais unidades, visando à assistência ao paciente no campo dos medicamentos e produtos de saúde, de forma segura e racional. Não importa o tamanho e a complexidade do hospital, é fato que, sem o medicamento e os correlatos não haverá sucesso na assistência sanitária ao paciente, os medicamentos representam uma quantia muito alta do orçamento dos hospitais, fazendo com isso um uso racional dos mesmos.

A farmácia deve ser considerada como um serviço clínico e hierarquicamente ligada à direção do hospital e ao serviço médico, e não aos serviços de administração de materiais e patrimônios, mais conhecida como almoxarifado, como ocorre usualmente, ela deve estar localizada em área de livre acesso e circulação, pois dessa forma facilita o atendimento à distribuição de medicamentos aos pacientes internados e ambulatoriais, como para receber dos fornecedores estes e demais produtos, também tem que dispor de um espaço suficiente para o desenvolvimento das diferentes atividades.

Toda farmácia de hospital, deve dispor de pelo menos algumas áreas consideradas essenciais, como a Central de Abastecimento Farmacêutico - CAF, área de dispensação interna e outras. Existe a função de compras na área de farmácia hospitalar, onde este é muito essencial, pois sua finalidade é suprir as necessidades de materiais e medicamentos e ter todo planejamento de quantidades para que assim sejam adquiridos na quantidade correta. Segundo Cavallini e Bisson (2002, p. 90), "[...] Comprar bem é um dos principais meios para redução dos custos hospitalares [...]".

Os insumos hospitalares e os medicamentos estocados nas farmácias possuem um custo muito elevado. Sabe-se que no setor da saúde, principalmente em hospitais, os recursos estão cada vez mais escassos, o que obriga aos gestores desses estabelecimentos uma busca por novas metodologias de controle. Em uma organização hospitalar, a prática de medicação pode ser definida como um sistema complexo, multidisciplinar, com vários processos interligados e interdependentes, constituída por profissionais de diferentes áreas como farmacêuticos, enfermeiros, médicos e auxiliares técnicos, onde estes profissionais compartilham um objetivo comum de prestação de assistência à saúde aos pacientes com eficiência, qualidade e segurança. 


\section{DISTRIBUIÇÃO DE MEDICAMENTOS}

É dever das três esferas governamentais a distribuição de medicamentos a toda população, independentemente do poder aquisitivo, considerando então que o acesso aos serviços de saúde deve ser universal e igualitário.

O processo de distribuição de medicamentos autoriza certificar uma utilização segura, eficaz e racional do medicamento realizado em estreita ligação com os serviços do hospital quer para o internamento quer para o serviço de ambulatório.

A Política Nacional de Medicamentos (PNM) está regulada pela Portaria n 3.916/98, do Ministério da Saúde, que estabelece critérios para aquisição, distribuição e entrega de medicamentos.

Todas as pessoas têm direito a receber medicamentos do Poder Público sem a necessidade de pagamento imediato. O art. $6^{\circ}$, inc. I alínea "d" da Lei $n^{\circ} 8.080 / 90$, inclui no âmbito de atuação do SUS a prestação de assistência farmacêutica.

Os medicamentos a qual todos têm direito pelo Poder Público têm que estar constando na relação da RENAME. Para isso todos os municípios têm que, com base na RENAME e no perfil da doença de sua população, elaborar uma relação de medicamentos essenciais para assim disponibilizar a todos os munícipes.

Estes medicamentos constantes da RENAME devem estar disponíveis a todos os brasileiros ou estrangeiros residentes no Brasil que deles necessitam.

Caso o medicamento necessitado não esteja constando na relação do município, mas tenha disponível na RENAME, é dever da União fazer a entrega do mesmo.

Em 2004, o Presidente da República, Luiz Inácio Lula da Silva, decretou e sancionou a Lei no 10.858, autorizando a Fundação Oswaldo Cruz - FIOCRUZ a disponibilizar medicamentos mediante ressarcimento, para custos de produção ou aquisição, distribuição e dispensação; no Art. $3^{\circ}$ desta Lei diz que a FIOCRUZ poderá firmar convênios com a União, Estados e Municípios, para que estes disponibilizem os medicamentos à população.

Já em 2009, foi publicado no Diário Oficial da União (de 16/04/09, Seção I - págs. 37 a 39) a Portaria GM no 749, onde dispõe sobre a expansão do Programa Farmácia Popular do Brasil - "Aqui Tem Farmácia Popular", no que diz respeito à distribuição dos medicamentos através do comércio varejista farmacêutico, onde estes medicamentos são pagos pelo Ministério da Saúde e sua complementação pelo usuário (paciente) de acordo com a tabela de valores e percentuais definidos no Anexo III desta Portaria. Para que se tenha o fornecimento através do comércio varejista o Ministério da Saúde tem que firmar uma parceria com o mesmo, a qual os medicamentos são definidos pelo o mesmo. Para que os estabelecimentos comerciais possam aderir ao Programa, tem que seguir o que exige o Art. $5^{\circ}$, Cap. II do Anexo III desta Portaria.

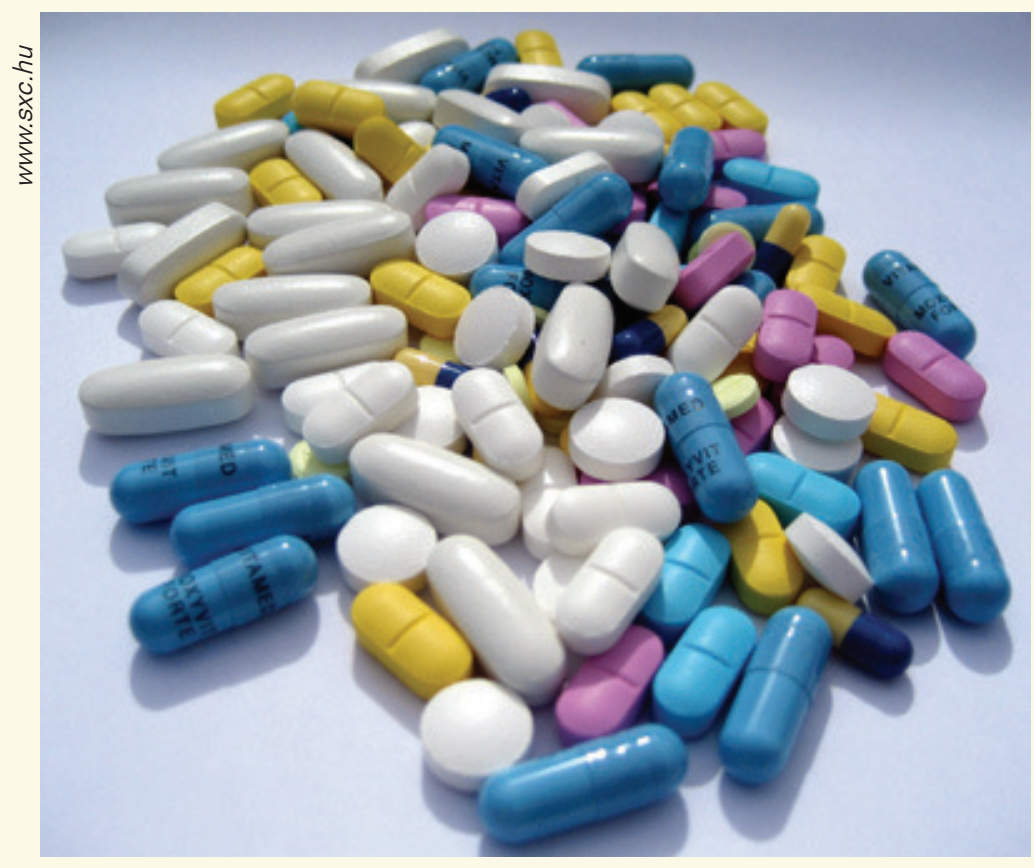

Os Decretos-Leis no 135/95, de 09 de junho, e 184/97, de 26 de julho, referentes aos medicamentos de uso humano e veterinário, respectivamente, determinam que os laboratórios/distribuidores autorizados a exercer as atividades de distribuição de medicamentos ficam obrigados a cumprir os princípios e normas das boas práticas de distribuição, onde segue esta norma na Portaria n 348/98.

Logo, percebe-se que a distribuição dos medicamentos ocorre de acordo com as legislações vigentes no Brasil, e desta forma todos os pacientes passaram a ter acesso gratuito, garantindo seu tratamento eficaz para obtenção da cura ou mesmo da sua sobrevida. 


\section{DISTRIBUIÇÃO DE MEDICAMENTOS PARA OS PACIENTES INTERNOS E EXTERNOS}

O artigo 196 da Constituição Federal assegura a todos o direito à vida e à saúde, representa consequência constitucional indissociável desse direito. A saúde é direito de todos e dever do Estado, garantido mediante políticas sociais e econômicas que visem à redução do risco de doença e de outros agravos e ao acesso universal igualitário às ações e serviços para sua promoção, proteção e recuperação.

A distribuição de medicamentos para os pacientes é feita de duas formas: uma é a distribuição por dose única e a outra é a distribuição geral. A distribuição por dose única é a feita no hospital ao paciente internado e que recebe o medicamento por dosagem e no horário indicado na prescrição médica; já a distribuição geral é feita para os pacientes externos que fazem a consulta e recebe o medicamento prescrito pelo médico para o tratamento por um mês ou até mesmo por mais tempo, onde estes medicamentos estão disponíveis nas farmácias públicas.

Segundo Rosa; Gomes; Reis (2003) o método de distribuição de medicamentos que oferece melhores condições para um adequado seguimento de terapia de medicamentos para pacientes é o Sistema de Distribuição de Medicamentos por Dose Unitária. O problema da implementação desse sistema é seu alto custo na implantação, que envolve máquinas, infraestrutura e número de funcionários adequados, mas esse investimento é compensado pela redução de custos que pode variar de 25\% a 40\%, em geral, e principalmente, redução com medicamentos e materiais.

Desta forma salienta-se saber que, o método de dose única é de grande importância para os hospitais, mesmo tendo um gasto alto na sua implantação, mas depois de toda estabilidade do método aplicado, vem à recompensa que é o de redução de custos.

De acordo com Cavallini e Bisson, (2002) um sistema de distribuição de medicamentos deve ser: racional, eficiente, econômico, seguro e deve estar de acordo com a terapêutica prescrita. Quanto maior a eficácia do sistema de distribuição mais garantido será o sucesso da terapêutica instaurada no hospital.

No dia 03 de fevereiro de 2011, durante o lançamento da campanha do Programa "Saúde Não Tem Preço", a Presidenta da República Dilma Rouseff anunciou a gratuidade de medicamentos para a população diabética e hipertensa, e estes medicamentos estão disponíveis nas farmácias comerciais que estão com o selo Aqui Tem Farmácia Popular, estas farmácias credenciadas que descumprirem os dispositivos da Portaria $n^{\circ} 184 / 11$, sofrerão penalidades e serão descredenciadas do programa.

Portanto, para o paciente que se encontra internado, o medicamento é utilizado em dose única. Já para os externos a obtenção dos mesmos se dá através de requisição médica e retira na farmácia do próprio hospital ou, na ausência deles, o paciente poderá adquirir através de farmácias credenciadas. Isto se faz necessário para que seja cumprida a lei, que diz: a saúde é direito de todos e dever do Estado.

\section{CONCLUSÃO}

A farmácia hospitalar é fundamental para toda a população brasileira, pois é através dela que se obtêm os medicamentos necessários para provimento da saúde pública. Apesar de ainda existir muita dificuldade para os pacientes adquirirem seus medicamentos em virtude da ineficiência do sistema de distribuição.

Ainda há muito para ser melhorado no gerenciamento e na distribuição dos medicamentos através dos órgãos públicos, pois a demanda aumenta e o suprimento dos medicamentos não acompanha o crescimento populacional, nem a evolução da ciência.

Portanto, cabe ao administrador executar as tarefas de planejamento, controle e uma série de requisitos favoráveis a uma boa administração da farmácia hospitalar. Atualmente ainda há muito para ser melhorado no gerenciamento e na distribuição dos medicamentos através dos órgãos públicos aos pacientes que deles necessitam. 


\section{8 - REFERÊNCIAS}

BRASIL. Constituição da República Federativa do Brasil de 1988. Emendas Constitucionais. Brasília, 05 de outubro de 1988. Disponível em: < http://www.planalto.gov.br/ccivil_03/constituicao/ constitui\%C3\%A7ao.htm>. Acesso em: 06/09/11.

BRASIL. Lei n 10858, de 13 de abril de 2004. Autoriza a Fundação Oswaldo Cruz-Fiocruz a disponibilizar medicamentos, mediante ressarcimento, e dá outras providências. Brasília, 13 de abril de 2004.

BRASIL. Lei n 9787, de 10 de fevereiro de 1999. Altera a Lei n 6360. Brasília, 10 de fevereiro de 1999. Disponível em: <http://www.planalto.gov.br/civil_03/leis//9787.htm>. Acesso em: 26/08/11.

BRASIL. Ministério da Saúde. Política Nacional de Medicamentos. Secretaria de Políticas de Saúde, Departamento de Atenção Básica. - Brasília: Ministério da Saúde, 2001.

BRASIL. Ministério da Saúde, Secretaria de Assistência à Saúde. Guia Básico para a Farmácia Hospitalar. Brasília/DF, 2004.

BRASIL. Ministério da Saúde, Secretaria de Ciência, Tecnologia e Insumos Estratégicos. Aquisição de Medicamentos para Assistência Farmacêutica no SUS. Brasília/DF, 2006.

BRASIL. Portaria $n^{\circ}$ 348, de 15 de junho de 1998. Dispõe sobre boas práticas de distribuição de medicamentos de usos humano e medicamentos veterinários. Disponível em: <http://www. infarmed.pt/portal/page/portal/INFARMED/LEGISLACAO/LEGISLACAO_FARMACEUTICA_ COMPILADA/TITULO_II/TITULO_IICAPITULO_III/portaria_348-98.pdf > . Acesso em 04/09/11

CAVALLINI, Míriam Elias; BISSON, Marcelo Polacow. Farmácia Hospitalar um enfoque em sistema de saúde. São Paulo: Manole, 2002.

EICKHOFF, P; HEINECK, I; SEIXAS, LJ. Gerenciamento e destinação final de medicamentos: uma discussão sobre o problema. Rev. BrRasev. F. Barmas, F 90 a (r1m), 6940-(618), 2009.

GONÇALVES, EL. (org.) Gestão Hospitalar: Administrando um hospital moderno. São Paulo: Saraiva, 2006.

MAXIMIANO, Antônio Cesar Amaru. Introdução à Administração. São Paulo: Atlas, 2007.

MINISTERIO DA SAÚDE. Lei $n^{\circ}$ 9.787, de 10 de fevereiro de 1999. Altera a Lei $n^{\circ} 6.360$, de 23 de setembro de 1976, que dispõe sobre a vigilância sanitária, estabelece o medicamento genérico, dispõe sobre a utilização de nomes genéricos em produtos farmacêuticos e dá outras providências.

MINISTERIO DA SAÚDE. Portaria n 3.916, em 30 de outubro de 1998, que "Dispõe sobre a aprovação da Política Nacional de Medicamentos e dá outras providências.

MINISTÉRIO DA SAÚDE. Portaria n 4.283, de 30 de dezembro de 2010. Diário Oficial da União [da] República Federativa do Brasil, Brasília, DF, 31 dez. 2010, Seção 1, p. 94-95.

ROSA, MB; GOMES, MJVM.; REIS, AMM. Abastecimento e Gerenciamento de Materiais. In: GOMES, Maria J. V. M.; REIS, Adriano M. M. Ciências Farmacêuticas: uma abordagem em farmácia hospitalar. 1. Ed. São Paulo: Atheneus. Cap. 21, p. 365-386. 2003.

SIMONETTI, V.M.M.; NOVAES, M.I.O; AFONSO, M.W. Gestão de suprimentos da farmácia hospitalar com a implantação de métodos gerenciais de insumos utilizados na manufatura. Revista Eletrônica Produção Engenharia. v.2 n.1 p. 57-68. Jan./Jul, 2009.

SILVA-NETA, FA; BRANCO, ES; LEITE, GA. Gerenciamento de medicamentos em redes hospitalares do Sistema Único de Saúde (SUS). Revista de Administração Hospitalar e Inovação em Saúde. p. 11-21. jan./jun. 2011. 